Acta Crystallographica Section E

Structure Reports

Online

ISSN 1600-5368

\section{(4S,5S)-2-(4-Chlorophenyl)-1,3- dioxolane-4,5-dicarboxamide}

\section{De-Cai Wang,* Jing Bai, Wei Xu, Tao Gai and Hua-Quan Liu}

School of Pharmaceutical Sciences, Nanjing University of Technology, Xinmofan Road No.5 Nanjing, Nanjing 210009, People's Republic of China

Correspondence e-mail: dcwang@njut.edu.cn

Received 13 June 2009; accepted 13 July 2009

Key indicators: single-crystal X-ray study; $T=293 \mathrm{~K}$; mean $\sigma(\mathrm{C}-\mathrm{C})=0.005 \AA$; $R$ factor $=0.048 ; w R$ factor $=0.122 ;$ data-to-parameter ratio $=11.8$.

The title compound, $\mathrm{C}_{11} \mathrm{H}_{11} \mathrm{ClN}_{2} \mathrm{O}_{4}$, is an important intermediate for the preparation of platinum anticancer drugs. The dioxolane ring adopts a twist conformation with an equatorially attached chlorophenyl substituent. In the crystal structure, molecules are linked into a two-dimensional network parallel to (001) by $\mathrm{N}-\mathrm{H} \cdots \mathrm{O}$ and $\mathrm{C}-\mathrm{H} \cdots \mathrm{O}$ hydrogen bonds.

\section{Related literature}

For bond-length data, see: Allen et al. (1987). For general background to platinum anticancer drugs, see: Kim et al. (1994); Pandey et al. (1997).<smiles>NC(=O)[C@H]1OC(c2ccc(Cl)cc2)O[C@@H]1C(N)=O</smiles>

\section{Experimental}

Crystal data

$\mathrm{C}_{11} \mathrm{H}_{11} \mathrm{ClN}_{2} \mathrm{O}_{4}$

$M_{r}=270.67$
Monoclinic, $P 2$

$$
\begin{aligned}
& a=9.2780(19) \AA \\
& b=4.7600(10) \AA \\
& c=13.245(3) \AA
\end{aligned}
$$

$\beta=93.15(3)^{\circ}$

$V=584.1(2) \AA^{3}$

$\mu=0.34 \mathrm{~mm}^{-1}$

$T=293 \mathrm{~K}$

$0.20 \times 0.20 \times 0.10 \mathrm{~mm}$

Mo $K \alpha$ radiation

Data collection

Enraf-Nonius CAD-4 diffractometer

Absorption correction: $\psi$ scan (North et al., 1968)

$T_{\min }=0.936, T_{\max }=0.967$

2248 measured reflections

Refinement

$R\left[F^{2}>2 \sigma\left(F^{2}\right)\right]=0.048$

$w R\left(F^{2}\right)=0.122$

$S=1.07$

2113 reflections

179 parameters

1 restraint

\author{
2113 independent reflections \\ 1694 reflections with $I>2 \sigma(I)$ \\ $R_{\text {int }}=0.027$ \\ 3 standard reflections \\ every 200 reflections \\ intensity decay: $1 \%$
}

Table 1

Hydrogen-bond geometry $\left(\AA,{ }^{\circ}\right)$.

\begin{tabular}{lllll}
\hline$D-\mathrm{H} \cdots A$ & $D-\mathrm{H}$ & $\mathrm{H} \cdots A$ & $D \cdots A$ & $D-\mathrm{H} \cdots A$ \\
\hline $\mathrm{N} 1-\mathrm{H} 1 A \cdots \mathrm{O} 3^{\mathrm{i}}$ & $0.79(4)$ & $2.20(4)$ & $2.979(5)$ & $166(4)$ \\
$\mathrm{N} 2-\mathrm{H} 2 A \cdots \mathrm{O} 3^{\text {ii }}$ & $0.87(4)$ & $2.42(3)$ & $3.173(5)$ & $145(3)$ \\
$\mathrm{N} 2-\mathrm{H} 2 B \cdots \mathrm{O} 4^{\text {iii }}$ & $0.81(6)$ & $2.26(6)$ & $3.012(4)$ & $155(4)$ \\
$\mathrm{C} 9-\mathrm{H} 9 \cdots \mathrm{O} 4^{\text {iii }}$ & 0.98 & 2.31 & $3.075(4)$ & 135 \\
\hline
\end{tabular}

Symmetry codes: (i) $-x+1, y-\frac{1}{2},-z+1$; (ii) $-x, y+\frac{1}{2},-z+1$; (iii) $x, y-1, z$.

Data collection: CAD-4 EXPRESS (Enraf-Nonius, 1989); cell refinement: CAD-4 EXPRESS; data reduction: XCAD4 (Harms \& Wocadlo, 1995); program(s) used to solve structure: SHELXS97 (Sheldrick, 2008); program(s) used to refine structure: SHELXL97 (Sheldrick, 2008); molecular graphics: SHELXTL (Sheldrick, 2008); software used to prepare material for publication: SHELXL97.

The authors thank the Center for Testing and Analysis, Nanjing University, for support.

Supplementary data and figures for this paper are available from the IUCr electronic archives (Reference: CI2828).

\title{
References
}

Allen, F. H., Kennard, O., Watson, D. G., Brammer, L., Orpen, A. G. \& Taylor, R. (1987). J. Chem. Soc. Perkin Trans. 2, pp. S1-19.

Enraf-Nonius (1989). CAD-4 EXPRESS. Enraf-Nonius, Delft, The Netherlands.

Flack, H. D. (1983). Acta Cryst. A39, 876-881.

Harms, K. \& Wocadlo, S. (1995). XCAD4. University of Marburg, Germany.

Kim, D. K., Kim, G., Gam, J., Cho, Y. B., Kim, H. T., Tai, J. H., Kim, K. H., Hong, W. S. \& Park, J. G. (1994). J. Med. Chem. 37, 1471-1485.

North, A. C. T., Phillips, D. C. \& Mathews, F. S. (1968). Acta Cryst. A24, 351359.

Pandey, G., Hajara, S., Ghorai, M. K. \& Kumar, K. R. (1997). J. Org. Chem. 62, 5966-5973.

Sheldrick, G. M. (2008). Acta Cryst. A64, 112-122. 


\section{supporting information}

Acta Cryst. (2009). E65, o1960 [doi:10.1107/S1600536809027494]

\section{(4S,5S)-2-(4-Chlorophenyl)-1,3-dioxolane-4,5-dicarboxamide}

\section{De-Cai Wang, Jing Bai, Wei Xu, Tao Gai and Hua-Quan Liu}

\section{S1. Comment}

Platinum antitumor drug is one kind of the most effective anticancer agents currently available. (2S,3S)-Diethyl 2,3-Oalkyltartrate analogues are starting materials for the syntheses of platinum complexes with antitumor activity (Kim $e t$ al.,1994), and are also important intermediates in organic syntheses (Pandey et al., 1997). As part of our studies on the syntheses and characterizations of these compounds, we have synthesized the title compound and reported herein its crystal structure.

In the molecule of the title compound (Fig 1), the bond lengths (Allen et al., 1987) and angles are within normal ranges. The dioxolane ring adopts a twist conformation and the chlorophenyl unit is equatorially attached.

In the crystal structure, $\mathrm{N}-\mathrm{H} \cdots \mathrm{O}$ and $\mathrm{C}-\mathrm{H} \cdots \mathrm{O}$ intermolecular hydrogen bonds (Table 1 ) link the molecules to form a two-dimensional network (Fig. 2) parallel to the (001).

\section{S2. Experimental}

4-Chlorobenzaldehyde (278 mg, $1.98 \mathrm{mmol}),(2 \mathrm{~S}, 3 \mathrm{~S})$-diethyltartrate ( $378 \mathrm{mg}, 1.84 \mathrm{mmol}$ ) and cyclohexane (10 ml) were placed in a round-bottomed flask, and 4-methylbenzenesulfonic acid $(30 \mathrm{mg})$ was added. The flask was fitted with a water-distributor. The mixture was heated under reflux for $3 \mathrm{~h}$. The reaction mixture was cooled to room temperature, and then transfered into a separatory funnel, washed with water $(200 \mathrm{ml})$ and extracted with acetate $(200 \mathrm{ml})$. The organic phase was distilled under pressure, and the residual was dissolved in anhydrous ethanol $(50 \mathrm{ml})$. Then, a current of dry ammonia was passed through the reaction mixture at room temperature for about $4 \mathrm{~h}$. The reaction mixture was then added dropwise to a vigorously stirred water $(600 \mathrm{ml})$. The resulting colourless precipitate was obtained by filtration and dried in vacuo (Kim et al., 1994). Single crystals suitable for X-ray analysis were obtained by slow evaporation of a methanol solution after two weeks.

\section{S3. Refinement}

$\mathrm{H}$ atoms were positioned geometrically $(\mathrm{C}-\mathrm{H}=0.93-0.97 \AA)$ and included in the refinement in riding motion approximation, with $U_{\text {iso }}(\mathrm{H})=1.2$ or $1.5 U_{\text {eq }}$ of the carrier atom. 


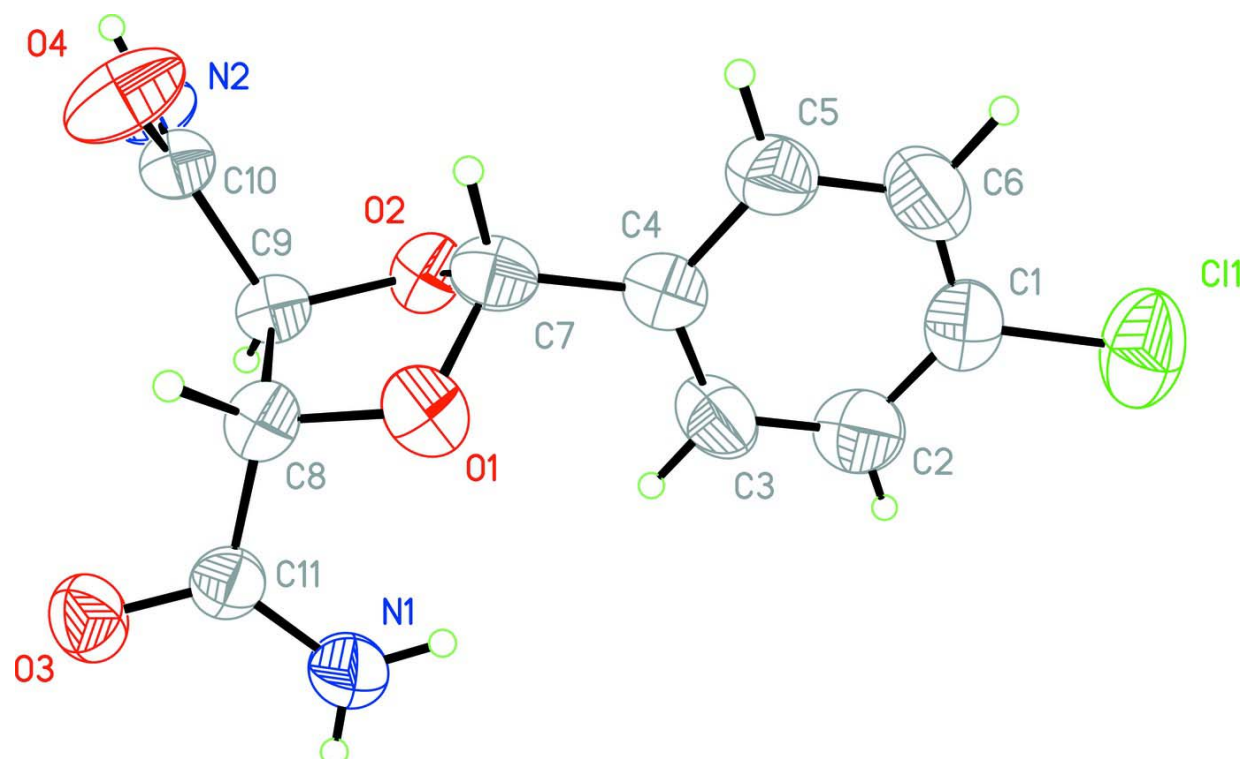

\section{Figure 1}

The molecular structure of the title compound, showing the atom-numbering scheme and displacement ellipsoids at the $30 \%$ probability level.

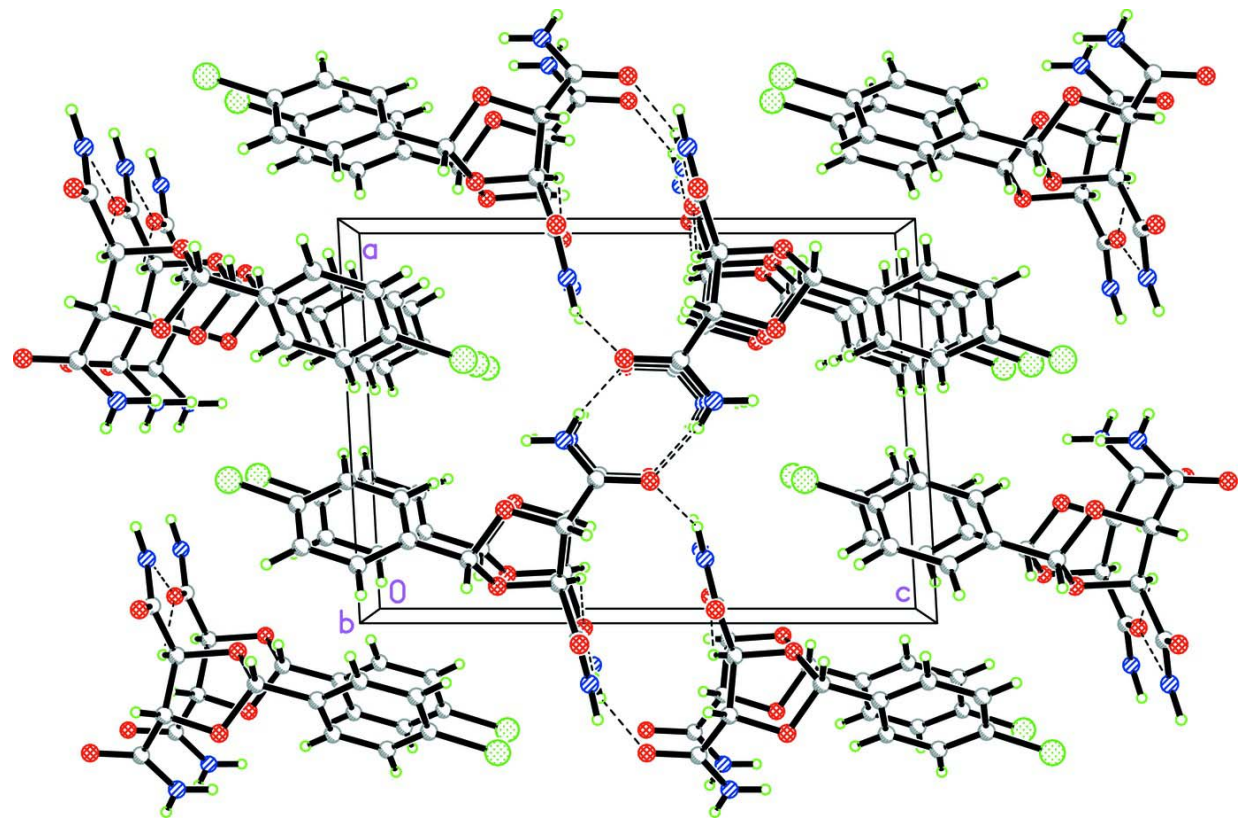

Figure 2

A packing diagram of the title compound. Hydrogen bonds are shown as dashed lines.

\section{(4S,5S)-2-(4-Chlorophenyl)-1,3-dioxolane-4,5-dicarboxamide}

Crystal data

$\mathrm{C}_{11} \mathrm{H}_{11} \mathrm{ClN}_{2} \mathrm{O}_{4}$

$M_{r}=270.67$

Monoclinic, $P 2_{1}$

Hall symbol: P 2yb

$$
\begin{aligned}
& a=9.2780(19) \AA \\
& b=4.760(1) \AA \\
& c=13.245(3) \AA \\
& \beta=93.15(3)^{\circ}
\end{aligned}
$$




$$
\begin{aligned}
& V=584.1(2) \AA^{3} \\
& Z=2 \\
& F(000)=280 \\
& D_{\mathrm{x}}=1.539 \mathrm{Mg} \mathrm{m}^{-3} \\
& \text { Mo } K \alpha \text { radiation, } \lambda=0.7 \\
& \text { Cell parameters from } 25 \\
& \text { Data collection } \\
& \text { Enraf-Nonius CAD-4 } \\
& \quad \text { diffractometer }
\end{aligned}
$$

\section{Data collection}$$
\text { Mo } K \alpha \text { radiation, } \lambda=0.71073 \AA
$$

Cell parameters from 25 reflections

Radiation source: fine-focus sealed tube Graphite monochromator $\omega / 2 \theta$ scans

Absorption correction: $\psi$ scan

(North et al., 1968)

$T_{\min }=0.936, T_{\max }=0.967$

2248 measured reflections

\section{Refinement}

Refinement on $F^{2}$

Least-squares matrix: full

$R\left[F^{2}>2 \sigma\left(F^{2}\right)\right]=0.048$

$w R\left(F^{2}\right)=0.122$

$S=1.07$

2113 reflections

179 parameters

1 restraint

Primary atom site location: structure-invariant direct methods

Secondary atom site location: difference Fourier map

$$
\begin{aligned}
& \theta=9-13^{\circ} \\
& \mu=0.34 \mathrm{~mm}^{-1} \\
& T=293 \mathrm{~K} \\
& \text { Block, colourless } \\
& 0.20 \times 0.20 \times 0.10 \mathrm{~mm}
\end{aligned}
$$

2113 independent reflections

1694 reflections with $I>2 \sigma(I)$

$R_{\text {int }}=0.027$

$\theta_{\max }=25.3^{\circ}, \theta_{\min }=1.5^{\circ}$

$h=0 \rightarrow 11$

$k=-5 \rightarrow 5$

$l=-15 \rightarrow 15$

3 standard reflections every 200 reflections intensity decay: $1 \%$

Hydrogen site location: inferred from neighbouring sites

$\mathrm{H}$ atoms treated by a mixture of independent and constrained refinement

$w=1 /\left[\sigma^{2}\left(F_{\mathrm{o}}^{2}\right)+(0.0601 P)^{2}+0.0558 P\right]$ where $P=\left(F_{\mathrm{o}}^{2}+2 F_{\mathrm{c}}^{2}\right) / 3$

$(\Delta / \sigma)_{\max }=0.001$

$\Delta \rho_{\max }=0.20 \mathrm{e}^{-3}$

$\Delta \rho_{\min }=-0.27$ e $\AA^{-3}$

Absolute structure: Flack (1983), 916 Friedel pairs

Absolute structure parameter: 0.07 (14)

\section{Special details}

Geometry. All e.s.d.'s (except the e.s.d. in the dihedral angle between two l.s. planes) are estimated using the full covariance matrix. The cell e.s.d.'s are taken into account individually in the estimation of e.s.d.'s in distances, angles and torsion angles; correlations between e.s.d.'s in cell parameters are only used when they are defined by crystal symmetry. An approximate (isotropic) treatment of cell e.s.d.'s is used for estimating e.s.d.'s involving 1.s. planes.

Refinement. Refinement of $F^{2}$ against ALL reflections. The weighted $R$-factor $w R$ and goodness of fit $S$ are based on $F^{2}$, conventional $R$-factors $R$ are based on $F$, with $F$ set to zero for negative $F^{2}$. The threshold expression of $F^{2}>\sigma\left(F^{2}\right)$ is used only for calculating $R$-factors (gt) etc. and is not relevant to the choice of reflections for refinement. $R$-factors based on $F^{2}$ are statistically about twice as large as those based on $F$, and $R$ - factors based on ALL data will be even larger.

Fractional atomic coordinates and isotropic or equivalent isotropic displacement parameters $\left(\AA^{2}\right)$

\begin{tabular}{lllll}
\hline & $x$ & $y$ & $z$ & $U_{\text {iso }} * / U_{\text {eq }}$ \\
\hline C11 & $0.35368(14)$ & $-0.0236(4)$ & $-0.21514(8)$ & $0.0925(5)$ \\
O1 & $0.2854(2)$ & $0.2859(5)$ & $0.26692(18)$ & $0.0475(6)$ \\
O2 & $0.0917(2)$ & $0.0071(5)$ & $0.24181(16)$ & $0.0461(6)$ \\
O3 & $0.3515(3)$ & $0.0218(6)$ & $0.51614(17)$ & $0.0568(7)$ \\
O4 & $-0.0323(3)$ & $0.4727(5)$ & $0.3827(2)$ & $0.0657(8)$ \\
N1 & $0.4522(4)$ & $-0.0804(9)$ & $0.3700(3)$ & $0.0597(10)$ \\
H1A & $0.516(4)$ & $-0.168(8)$ & $0.397(3)$ & $0.038(10)^{*}$ \\
H1B & $0.451(4)$ & $-0.069(10)$ & $0.305(3)$ & $0.064(13)^{*}$
\end{tabular}




$\begin{array}{lllll}\mathrm{N} 2 & -0.1472(4) & 0.0634(7) & 0.3925(3) & 0.0458(7) \\ \mathrm{H} 2 \mathrm{~A} & -0.230(4) & 0.139(7) & 0.403(2) & 0.034(9)^{*} \\ \mathrm{H} 2 \mathrm{~B} & -0.140(5) & -0.105(12) & 0.399(3) & 0.071(15)^{*} \\ \mathrm{C} 1 & 0.2948(4) & 0.0470(10) & -0.0955(3) & 0.0573(10) \\ \mathrm{C} 2 & 0.3508(5) & -0.1037(10) & -0.0134(3) & 0.0675(12) \\ \mathrm{H} 2 & 0.4179 & -0.2455 & -0.0224 & 0.081^{*} \\ \mathrm{C} 3 & 0.3072(4) & -0.0438(10) & 0.0813(3) & 0.0618(10) \\ \mathrm{H} 3 & 0.3440 & -0.1476 & 0.1363 & 0.074^{*} \\ \mathrm{C} 4 & 0.2085(4) & 0.1701(7) & 0.0964(3) & 0.0467(9) \\ \mathrm{C} 5 & 0.1527(4) & 0.3126(9) & 0.0133(3) & 0.0614(11) \\ \mathrm{H} 5 & 0.0852 & 0.4541 & 0.0216 & 0.074^{*} \\ \text { C6 } & 0.1949(5) & 0.2500(11) & -0.0830(3) & 0.0701(12) \\ \mathrm{H} 6 & 0.1549 & 0.3468 & -0.1387 & 0.084^{*} \\ \text { C7 } & 0.1632(4) & 0.2384(7) & 0.1998(3) & 0.0471(8) \\ \mathrm{H} 7 & 0.1002 & 0.4038 & 0.1976 & 0.057^{*} \\ \text { C8 } & 0.2436(3) & 0.2201(7) & 0.3666(2) & 0.0392(7) \\ \text { H8 } & 0.2264 & 0.3935 & 0.4039 & 0.047^{*} \\ \text { C9 } & 0.1005(3) & 0.0548(7) & 0.3490(2) & 0.0378(7) \\ \text { H9 } & 0.1064 & -0.1248 & 0.3852 & 0.045^{*} \\ \text { C10 } & -0.0327(3) & 0.2166(7) & 0.3777(3) & 0.0395(8) \\ \text { C11 } & 0.3560(3) & 0.0449(8) & 0.4236(2) & 0.0423(8) \\ \end{array}$

Atomic displacement parameters $\left(\AA^{2}\right)$

\begin{tabular}{lllllll}
\hline & $U^{11}$ & $U^{22}$ & $U^{33}$ & $U^{12}$ & $U^{13}$ & $U^{23}$ \\
\hline C11 & $0.1056(9)$ & $0.1139(10)$ & $0.0597(6)$ & $-0.0231(10)$ & $0.0197(6)$ & $-0.0196(7)$ \\
O1 & $0.0522(13)$ & $0.0425(14)$ & $0.0473(13)$ & $-0.0157(11)$ & $-0.0015(11)$ & $0.0055(11)$ \\
O2 & $0.0474(12)$ & $0.0375(13)$ & $0.0539(13)$ & $-0.0122(12)$ & $0.0066(10)$ & $-0.0116(12)$ \\
O3 & $0.0565(14)$ & $0.0719(19)$ & $0.0423(13)$ & $-0.0098(15)$ & $0.0051(11)$ & $0.0030(14)$ \\
O4 & $0.0582(15)$ & $0.0206(12)$ & $0.120(2)$ & $0.0031(12)$ & $0.0207(14)$ & $-0.0055(15)$ \\
N1 & $0.055(2)$ & $0.074(3)$ & $0.050(2)$ & $0.0199(19)$ & $-0.0040(17)$ & $-0.0013(18)$ \\
N2 & $0.0402(17)$ & $0.0289(17)$ & $0.069(2)$ & $0.0016(14)$ & $0.0126(14)$ & $0.0046(14)$ \\
C1 & $0.057(2)$ & $0.064(3)$ & $0.051(2)$ & $-0.019(2)$ & $0.0029(17)$ & $-0.008(2)$ \\
C2 & $0.071(3)$ & $0.065(3)$ & $0.066(3)$ & $0.011(2)$ & $-0.003(2)$ & $-0.019(2)$ \\
C3 & $0.078(3)$ & $0.058(2)$ & $0.048(2)$ & $0.012(2)$ & $-0.0093(18)$ & $-0.003(2)$ \\
C4 & $0.049(2)$ & $0.0377(19)$ & $0.053(2)$ & $-0.0036(16)$ & $-0.0039(17)$ & $-0.0016(16)$ \\
C5 & $0.057(2)$ & $0.061(3)$ & $0.066(3)$ & $0.009(2)$ & $0.0015(19)$ & $0.012(2)$ \\
C6 & $0.073(3)$ & $0.082(3)$ & $0.055(2)$ & $-0.005(3)$ & $-0.006(2)$ & $0.016(2)$ \\
C7 & $0.049(2)$ & $0.0352(18)$ & $0.057(2)$ & $0.0005(17)$ & $-0.0044(17)$ & $0.0031(16)$ \\
C8 & $0.0428(18)$ & $0.0303(17)$ & $0.0450(18)$ & $-0.0061(15)$ & $0.0073(15)$ & $-0.0031(14)$ \\
C9 & $0.0388(16)$ & $0.0245(15)$ & $0.0500(19)$ & $-0.0048(14)$ & $0.0021(14)$ & $-0.0002(13)$ \\
C10 & $0.0395(17)$ & $0.0312(19)$ & $0.0479(19)$ & $-0.0016(15)$ & $0.0023(15)$ & $0.0005(15)$ \\
C11 & $0.0343(16)$ & $0.045(2)$ & $0.0475(19)$ & $-0.0100(16)$ & $0.0032(14)$ & $-0.0074(17)$ \\
& & & & & & \\
\hline
\end{tabular}

Geometric parameters $\left(\AA,{ }^{\circ}\right)$

\begin{tabular}{llll}
\hline $\mathrm{C} 11-\mathrm{C} 1$ & $1.738(4)$ & $\mathrm{C} 2-\mathrm{C} 3$ & $1.370(5)$ \\
$\mathrm{O} 1-\mathrm{C} 7$ & $1.420(4)$ & $\mathrm{C} 2-\mathrm{H} 2$ & 0.93
\end{tabular}




\begin{tabular}{|c|c|c|c|}
\hline $\mathrm{O} 1-\mathrm{C} 8$ & $1.431(4)$ & $\mathrm{C} 3-\mathrm{C} 4$ & $1.391(5)$ \\
\hline $\mathrm{O} 2-\mathrm{C} 7$ & $1.415(4)$ & $\mathrm{C} 3-\mathrm{H} 3$ & 0.93 \\
\hline $\mathrm{O} 2-\mathrm{C} 9$ & $1.436(4)$ & $\mathrm{C} 4-\mathrm{C} 5$ & $1.370(5)$ \\
\hline $\mathrm{O} 3-\mathrm{C} 11$ & $1.233(4)$ & $\mathrm{C} 4-\mathrm{C} 7$ & $1.490(5)$ \\
\hline $\mathrm{O} 4-\mathrm{C} 10$ & $1.221(4)$ & $\mathrm{C} 5-\mathrm{C} 6$ & $1.387(6)$ \\
\hline $\mathrm{N} 1-\mathrm{C} 11$ & $1.313(5)$ & $\mathrm{C} 5-\mathrm{H} 5$ & 0.93 \\
\hline $\mathrm{N} 1-\mathrm{H} 1 \mathrm{~A}$ & $0.79(4)$ & $\mathrm{C} 6-\mathrm{H} 6$ & 0.93 \\
\hline N1-H1B & $0.86(4)$ & $\mathrm{C} 7-\mathrm{H} 7$ & 0.98 \\
\hline $\mathrm{N} 2-\mathrm{C} 10$ & $1.312(5)$ & $\mathrm{C} 8-\mathrm{C} 11$ & $1.506(5)$ \\
\hline $\mathrm{N} 2-\mathrm{H} 2 \mathrm{~A}$ & $0.86(3)$ & $\mathrm{C} 8-\mathrm{C} 9$ & $1.550(4)$ \\
\hline $\mathrm{N} 2-\mathrm{H} 2 \mathrm{~B}$ & $0.81(6)$ & $\mathrm{C} 8-\mathrm{H} 8$ & 0.98 \\
\hline $\mathrm{C} 1-\mathrm{C} 6$ & $1.355(6)$ & $\mathrm{C} 9-\mathrm{C} 10$ & $1.522(4)$ \\
\hline $\mathrm{C} 1-\mathrm{C} 2$ & $1.379(6)$ & C9- & 0.98 \\
\hline $\mathrm{C} 7-\mathrm{O} 1-\mathrm{C} 8$ & $107.2(2)$ & $\mathrm{C} 5-\mathrm{C} 6-\mathrm{H} 6$ & 120.2 \\
\hline $\mathrm{C} 7-\mathrm{O} 2-\mathrm{C} 9$ & $105.3(2)$ & $\mathrm{O} 2-\mathrm{C} 7-\mathrm{O} 1$ & $104.7(3)$ \\
\hline $\mathrm{C} 11-\mathrm{N} 1-\mathrm{H} 1 \mathrm{~A}$ & $120(3)$ & $\mathrm{O} 2-\mathrm{C} 7-\mathrm{C} 4$ & $110.7(3)$ \\
\hline $\mathrm{C} 11-\mathrm{N} 1-\mathrm{H} 1 \mathrm{~B}$ & $123(3)$ & $\mathrm{O} 1-\mathrm{C} 7-\mathrm{C} 4$ & $110.8(3)$ \\
\hline $\mathrm{H} 1 \mathrm{~A}-\mathrm{N} 1-\mathrm{H} 1 \mathrm{~B}$ & $117(4)$ & $\mathrm{O} 2-\mathrm{C} 7-\mathrm{H} 7$ & 110.2 \\
\hline $\mathrm{C} 10-\mathrm{N} 2-\mathrm{H} 2 \mathrm{~A}$ & $122(2)$ & $\mathrm{O} 1-\mathrm{C} 7-\mathrm{H} 7$ & 110.2 \\
\hline $\mathrm{C} 10-\mathrm{N} 2-\mathrm{H} 2 \mathrm{~B}$ & $121(3)$ & $\mathrm{C} 4-\mathrm{C} 7-\mathrm{H} 7$ & 110.2 \\
\hline $\mathrm{H} 2 \mathrm{~A}-\mathrm{N} 2-\mathrm{H} 2 \mathrm{~B}$ & $117(4)$ & $\mathrm{O} 1-\mathrm{C} 8-\mathrm{C} 11$ & $111.6(3)$ \\
\hline $\mathrm{C} 6-\mathrm{C} 1-\mathrm{C} 2$ & $120.4(4)$ & $\mathrm{O} 1-\mathrm{C} 8-\mathrm{C} 9$ & $104.2(2)$ \\
\hline $\mathrm{C} 6-\mathrm{C} 1-\mathrm{Cl1}$ & $120.0(3)$ & $\mathrm{C} 11-\mathrm{C} 8-\mathrm{C} 9$ & $111.0(3)$ \\
\hline $\mathrm{C} 2-\mathrm{C} 1-\mathrm{Cl1}$ & $119.6(3)$ & $\mathrm{O} 1-\mathrm{C} 8-\mathrm{H} 8$ & 109.9 \\
\hline $\mathrm{C} 3-\mathrm{C} 2-\mathrm{C} 1$ & $119.7(4)$ & $\mathrm{C} 11-\mathrm{C} 8-\mathrm{H} 8$ & 109.9 \\
\hline $\mathrm{C} 3-\mathrm{C} 2-\mathrm{H} 2$ & 120.1 & $\mathrm{C} 9-\mathrm{C} 8-\mathrm{H} 8$ & 109.9 \\
\hline $\mathrm{C} 1-\mathrm{C} 2-\mathrm{H} 2$ & 120.1 & $\mathrm{O} 2-\mathrm{C} 9-\mathrm{C} 10$ & $108.9(2)$ \\
\hline $\mathrm{C} 2-\mathrm{C} 3-\mathrm{C} 4$ & $121.0(4)$ & $\mathrm{O} 2-\mathrm{C} 9-\mathrm{C} 8$ & $103.3(2)$ \\
\hline $\mathrm{C} 2-\mathrm{C} 3-\mathrm{H} 3$ & 119.5 & $\mathrm{C} 10-\mathrm{C} 9-\mathrm{C} 8$ & $114.0(3)$ \\
\hline $\mathrm{C} 4-\mathrm{C} 3-\mathrm{H} 3$ & 119.5 & $\mathrm{O} 2-\mathrm{C} 9-\mathrm{H} 9$ & 110.1 \\
\hline $\mathrm{C} 5-\mathrm{C} 4-\mathrm{C} 3$ & $118.0(4)$ & $\mathrm{C} 10-\mathrm{C} 9-\mathrm{H} 9$ & 110.1 \\
\hline $\mathrm{C} 5-\mathrm{C} 4-\mathrm{C} 7$ & $121.2(3)$ & $\mathrm{C} 8-\mathrm{C} 9-\mathrm{H} 9$ & 110.1 \\
\hline $\mathrm{C} 3-\mathrm{C} 4-\mathrm{C} 7$ & $120.9(3)$ & $\mathrm{O} 4-\mathrm{C} 10-\mathrm{N} 2$ & $123.2(3)$ \\
\hline $\mathrm{C} 4-\mathrm{C} 5-\mathrm{C} 6$ & $121.3(4)$ & $\mathrm{O} 4-\mathrm{C} 10-\mathrm{C} 9$ & $121.2(3)$ \\
\hline $\mathrm{C} 4-\mathrm{C} 5-\mathrm{H} 5$ & 119.4 & $\mathrm{~N} 2-\mathrm{C} 10-\mathrm{C} 9$ & $115.5(3)$ \\
\hline $\mathrm{C} 6-\mathrm{C} 5-\mathrm{H} 5$ & 119.4 & $\mathrm{O} 3-\mathrm{C} 11-\mathrm{N} 1$ & $123.9(3)$ \\
\hline $\mathrm{C} 1-\mathrm{C} 6-\mathrm{C} 5$ & $119.7(4)$ & $\mathrm{O} 3-\mathrm{C} 11-\mathrm{C} 8$ & $119.2(3)$ \\
\hline $\mathrm{C} 1-\mathrm{C} 6-\mathrm{H} 6$ & 120.2 & $\mathrm{~N} 1-\mathrm{C} 11-\mathrm{C} 8$ & $116.9(3)$ \\
\hline $\mathrm{C} 6-\mathrm{C} 1-\mathrm{C} 2-\mathrm{C} 3$ & $-1.3(6)$ & $\mathrm{C} 3-\mathrm{C} 4-\mathrm{C} 7-\mathrm{O} 1$ & $52.9(5)$ \\
\hline $\mathrm{C} 11-\mathrm{C} 1-\mathrm{C} 2-\mathrm{C} 3$ & $178.4(3)$ & $\mathrm{C} 7-\mathrm{O} 1-\mathrm{C} 8-\mathrm{C} 11$ & $134.7(3)$ \\
\hline $\mathrm{C} 1-\mathrm{C} 2-\mathrm{C} 3-\mathrm{C} 4$ & $-0.9(7)$ & $\mathrm{C} 7-\mathrm{O} 1-\mathrm{C} 8-\mathrm{C} 9$ & $14.8(3)$ \\
\hline $\mathrm{C} 2-\mathrm{C} 3-\mathrm{C} 4-\mathrm{C} 5$ & $2.1(6)$ & $\mathrm{C} 7-\mathrm{O} 2-\mathrm{C} 9-\mathrm{C} 10$ & $91.5(3)$ \\
\hline $\mathrm{C} 2-\mathrm{C} 3-\mathrm{C} 4-\mathrm{C} 7$ & $-178.9(4)$ & $\mathrm{C} 7-\mathrm{O} 2-\mathrm{C} 9-\mathrm{C} 8$ & $-30.0(3)$ \\
\hline $\mathrm{C} 3-\mathrm{C} 4-\mathrm{C} 5-\mathrm{C} 6$ & $-1.2(6)$ & $\mathrm{O} 1-\mathrm{C} 8-\mathrm{C} 9-\mathrm{O} 2$ & $9.3(3)$ \\
\hline $\mathrm{C} 7-\mathrm{C} 4-\mathrm{C} 5-\mathrm{C} 6$ & $179.8(4)$ & $\mathrm{C} 11-\mathrm{C} 8-\mathrm{C} 9-\mathrm{O} 2$ & $-111.0(3)$ \\
\hline $\mathrm{C} 2-\mathrm{C} 1-\mathrm{C} 6-\mathrm{C} 5$ & $2.2(6)$ & $\mathrm{O} 1-\mathrm{C} 8-\mathrm{C} 9-\mathrm{C} 10$ & $-108.7(3)$ \\
\hline
\end{tabular}




$\begin{array}{llll}\mathrm{C} 11-\mathrm{C} 1-\mathrm{C} 6-\mathrm{C} 5 & -177.5(3) & \mathrm{C} 11-\mathrm{C} 8-\mathrm{C} 9-\mathrm{C} 10 & 130.9(3) \\ \mathrm{C} 4-\mathrm{C} 5-\mathrm{C} 6-\mathrm{C} 1 & -1.0(7) & \mathrm{O} 2-\mathrm{C} 9-\mathrm{C} 10-\mathrm{O} 4 & -93.1(4) \\ \mathrm{C} 9-\mathrm{O} 2-\mathrm{C} 7-\mathrm{O} 1 & 40.4(3) & \mathrm{C} 8-\mathrm{C} 9-\mathrm{C} 10-\mathrm{O} 4 & 21.7(5) \\ \mathrm{C} 9-\mathrm{O} 2-\mathrm{C} 7-\mathrm{C} 4 & 159.7(3) & \mathrm{O} 2-\mathrm{C} 9-\mathrm{C} 10-\mathrm{N} 2 & 84.4(4) \\ \mathrm{C} 8-\mathrm{O} 1-\mathrm{C} 7-\mathrm{O} 2 & -34.2(3) & \mathrm{C} 8-\mathrm{C} 9-\mathrm{C} 10-\mathrm{N} 2 & -160.8(3) \\ \mathrm{C} 8-\mathrm{O} 1-\mathrm{C} 7-\mathrm{C} 4 & -153.5(3) & \mathrm{O} 1-\mathrm{C} 8-\mathrm{C} 11-\mathrm{O} 3 & 164.5(3) \\ \mathrm{C} 5-\mathrm{C} 4-\mathrm{C} 7-\mathrm{O} 2 & 116.2(4) & \mathrm{C} 9-\mathrm{C} 8-\mathrm{C} 11-\mathrm{O} 3 & -79.7(4) \\ \mathrm{C} 3-\mathrm{C} 4-\mathrm{C} 7-\mathrm{O} 2 & -62.8(4) & \mathrm{O} 1-\mathrm{C} 8-\mathrm{C} 11-\mathrm{N} 1 & -17.0(4) \\ \mathrm{C} 5-\mathrm{C} 4-\mathrm{C} 7-\mathrm{O} 1 & -128.1(4) & \mathrm{C} 9-\mathrm{C} 8-\mathrm{C} 11-\mathrm{N} 1 & 98.8(4)\end{array}$

Hydrogen-bond geometry $\left(A,{ }^{\circ}\right)$

\begin{tabular}{lllll}
\hline$D-\mathrm{H} \cdots A$ & $D-\mathrm{H}$ & $\mathrm{H} \cdots A$ & $D \cdots A$ & $D-\mathrm{H} \cdots A$ \\
\hline $\mathrm{N} 1-\mathrm{H} 1 A^{\cdots} \cdots 3^{\mathrm{i}}$ & $0.79(4)$ & $2.20(4)$ & $2.979(5)$ & $166(4)$ \\
$\mathrm{N} 2-\mathrm{H} 2 A \cdots \mathrm{O} 3^{\text {ii }}$ & $0.87(4)$ & $2.42(3)$ & $3.173(5)$ & $145(3)$ \\
$\mathrm{N} 2-\mathrm{H} 2 B \cdots \mathrm{O} 4^{\mathrm{iii}}$ & $0.81(6)$ & $2.26(6)$ & $3.012(4)$ & $155(4)$ \\
$\mathrm{C} 9-\mathrm{H} 9 \cdots \mathrm{O} 4^{\mathrm{iii}}$ & 0.98 & 2.31 & $3.075(4)$ & 135
\end{tabular}

Symmetry codes: (i) $-x+1, y-1 / 2,-z+1$; (ii) $-x, y+1 / 2,-z+1$; (iii) $x, y-1, z$. 